\title{
Intervention of Cardiovascular Aging for Improving the Health Status of Elderly People
}

The recent global growth of the aging population strongly emphasizes the need to study common and important diseases affecting the elderly for disease prevention and promotion of the general health of these individuals. The incidence and prevalence of cardiovascular diseases are well known to increase steeply with advancing age. Furthermore, cardiovascular diseases and its risk factors are associated with functional impairment in older adults ${ }^{1)}$, warranting the development of strategies to control cardiovascular diseases for the continuance of independent functional status.

Although, cardiovascular changes with aging have been considered as a part of "normal" physiologic phenomenon, it is now believed as a distinct clinical manifestation that can predict a higher risk of developing atherosclerosis, hypertension, heart failure, and stroke. In other words, aging is one of the main risk factors for the development of cardiovascular diseases because of its association with complex structural and functional alterations in the cardiovascular system. Hence, in order to understand clinical presentations and pathophysiology of cardiovascular diseases in elderly patients, it is imperative to investigate age-associated changes in the cardiovascular structure and function ${ }^{2)}$.

One of the characteristic effects of aging is the stiffening of large artery or arteriosclerosis, which is increasingly being considered as an independent determinant of cardiovascular risk. Age-related changes in the arterial structure may be relevant in the development of isolated systolic hypertension in elderly patients. Understanding the mechanism underlying increased arterial stiffness would enable researchers to control vascular aging, and consequently, other cardiovascular disease conditions such as isolated systolic hypertension, heart failure, and atrial fibrillation ${ }^{3)}$. Interestingly, increased arterial stiffness has been reported in individuals with cardiovascular risk factors such as hypercholesterolemia, smoking, obesity, and diabetes mellitus, leading to the popular concept that such individuals may experience accelerated vascular aging compared to their healthier counterparts. Considering that, vascular aging has been associated with physical and cognitive functional decline in the elderly population; therapeutic intervention for vascular aging is needed to prevent functional decline in the elderly population.

Current evidences supported the role of antihypertensive therapies such as the renin-angiotensin-aldosterone system blockade with angiotensin converting enzyme inhibitors or angiotensin receptor blockers or calcium channel blockers or diuretics, in the lowering of arterial stiffness and subsequently decreasing systolic blood pressure. In contrast, beta-blocker has little effect on the increased arterial stiffness of the elderly people ${ }^{4)}$.

Earlier intervention is crucial owing to its effectiveness in harnessing the maximal benefits of the therapy, when compared to current treatment strategies. In other words, the phrase "the earlier, the better" should be highlighted for the prevention of cardiovascular aging. Finally, in contrast to currently available therapies, future novel treatments focusing on agespecific targets such as anti-inflammation and caloric restriction mimetics may provide a more effective reduction in pulse pressure, and hence are better able to prevent the development of cardiovascular disease in elderly patients ${ }^{5}$.

\section{Kwang-Il Kim \\ Editorial Board Member}

Department of Internal Medicine,
Seoul National University Bundang Hospital,
Seoul National University College of Medicine,
Seongnam, Korea

\section{REFERENCES}

1. Hajjar I, Lackland DT, Cupples LA, Lipsitz LA. Association between concurrent and remote blood pressure and disability in older adults. Hypertension 2007;50:1026-32.

2. Paneni F, Diaz Cañestro C, Libby P, Lüscher TF, Camici GG. The aging cardiovascular system: understanding it at the cellular and clinical levels. J Am Coll Cardiol 2017;69:1952-67.

3. Lakatta EG, Levy D. Arterial and cardiac aging: major shareholders in cardiovascular disease enterprises: Part I: aging arteries: a "set up" for vascular disease. Circulation 2003;107:139-46.

4. Kim KI, Cho YS, Choi DJ, Kim CH. Optimal treatment of hypertension in the elderly: a Korean perspective. Geriatr Gerontol Int 2008;8:5-11.

5. Ungvari Z, Kaley G, de Cabo R, Sonntag WE, Csiszar A. Mechanisms of vascular aging: new perspectives. J Gerontol A Biol Sci Med Sci 2010;65:1028-41. 